\title{
Evaluación curricular e investigación. Un recuento de lo hecho en el INEE de México
}

\section{Curricular evaluation and research. An account of the work conducted at Mexico's INEE}

\section{DOl: https://doi.org/10.32870/dse.v0i20.546}

\author{
Mario Alberto Benavides Lara* \\ Pamela Manzano Gutiérrez**
}

\begin{abstract}
Resumen
En este trabajo se presenta parte del ejercicio de sistematización conceptual que permitió a un grupo de funcionarios construir una propuesta práctica de evaluación del currículo basada en la recuperación de la lógica de la investigación educativa. Con esto se quiere demostrar el potencial que los enfoques cualitativos y cuantitativos de la metodología de investigación tienen para la evaluación del currículo, como parte de la política educativa de un país. A lo largo de este texto, se aborda el proceso de construcción metodológica que implicó definir un área de trabajo de un instituto de evaluación educativa en México, y cómo este trabajo se ha traducido en evaluaciones en torno a diferentes aspectos del currículo. Lo anterior permitirá al lector, especialmente a aquellos que se dediquen a evaluar el currículo, proyectar nuevos escenarios de trabajo en los que la innovación metodológica sea una característica.
\end{abstract}

Palabras clave: currículo - evaluación - diseño curricular - educación obligatoria - sistema educativo.

\begin{abstract}
This paper presents part of the conceptual systematization work that has allowed a group of officials to construct a practical proposal for the evaluation of the curriculum and which is based on the recovery of the logic of educational research, in order to demonstrate the potential that the qualitative and quantitative research methodology approaches have to evaluate the curricula as part of the educational policy of a country.

Throughout this text the process of methodological construction that involved defining a work area of an educational evaluation institute in Mexico is addressed, as well as how this work has resulted in evaluations around different aspects of the curricula in order to allow the reader, especially those who are tasked with the evaluation of the curricula, to project new work scenarios in which methodological innovation is a characteristic.
\end{abstract}

Keywords: curriculum - evaluation - curriculum design - compulsory education - educational system.

\footnotetext{
* Pedagogo. Líneas de trabajo: política educativa, enfoques didácticos y estudios sociales de la educación. México.mario.benavides@outlook. com

** Maestra en pedagogía. Dirección de evaluación de contenidos y métodos educativos. Línea de trabajo: currículo y evaluación. México.pmanzano@inee.edu.mx
} 


\section{Introducción}

El propósito de este trabajo es hacer una descripción analítica de la forma en que se articulan la investigación y la evaluación en la construcción del objeto de evaluación de la Dirección de Contenidos y Métodos Educativo, por sus siglas la DECME, del ahora extinto Instituto Nacional para la Evaluación Educativa (INEE), ${ }^{1}$ de México: el currículo.

Para ello se recurrirá a identificar cómo el proceso de construcción de las evaluaciones del currículo, en sus dimensiones de diseño e implementación, evoca el proceso de construcción de conocimiento propio de la investigación. Con esto se buscará dar cuenta de la indivisibilidad de ambos procesos — la investigación y la evaluación-, y a la vez delinear algunos de los retos que esto supone para la evaluación y que tienen que ver con: el alcance de los métodos cuantitativos frente a los cualitativos y la generalización de sus resultados; la delimitación de los constructos en un entorno organizativo como el del instituto; así como la amplitud de procesos que implica la evaluación del currículo.

La importancia de la evaluación del currículo radica en que se constituye como el principal orientador y prescriptor de las prácticas de aula, al mismo tiempo que se manifiesta como un organizador de los recursos y sentidos que adquieren las políticas educativas en las escuelas (INEE, 2016; 2019).

Considerando esta definición de currículo, su evaluación requiere modelos innovadores que permitan identificar, en el marco de una evaluación cuantitativa y a gran escala, los constructos más pertinentes para aproximarse a la comprensión de las prácticas en relación con el currículo y, en el caso de estudios de corte cualitativo, para profundizar en los significados que estas prácticas adquieren, atendiendo siempre las limitaciones propias del uso de instrumentos y metodologías específicas.

\section{La construcción de un objeto de investigación y evaluación}

En el análisis y evaluación de las políticas educativas es común ver a los componentes, procesos y resultados del Sistema Educativo Nacional (SEN) más como insumos que como procesos problemáticos a considerar en el diseño de la política educativa, sobre todo cuando se refieren a elementos de tipo pedagógico y didáctico. Se puede argüir que estas diferencias en torno a los procesos clave de la educación se deben a las racionalidades con las que distintas disciplinas concurrentes en la educación construyen su objeto de estudio y que derivan en usos diferentes de las metodologías de investigación que emplean para develar los procesos sobre los que indagan (Becher, 2009).

1 A partir de la reforma constitucional en materia educativa que entró en vigor el 15 de mayo de 2019 y en la que se derogaron diversas disposiciones de los artículos 3, 31 y 73 de la Constitución Política de los Estados Unidos Mexicanos, desapareció el Instituto Nacional para la Evaluación de la Educación y, con la promulgación de las leyes secundarias de dicha reforma, nació la Comisión Nacional para la Mejora Continua de la Educación, figura que sustituye al Instituto. Sin embargo, a lo largo del texto se hablará del Instituto por tratarse de la recuperación de experiencias acumuladas en el marco de su existencia y a propósito de las atribuciones que le fueron conferidas.

Diálo@os 
Aunque la noción de currículo es familiar para quienes nos dedicamos a la educación, y que, además, de acuerdo con Gimeno (2011) es un término cuya acuñación no supera los cuarenta años, no implica que la entendamos igual; aún cuando existe un punto de toque con la idea de que el currículo es un símil de los planes y programas de estudio, es insuficiente cuando se trata de explicarlo desde referentes más robustos que incorporan una posición epistemológica y ontológica, y que dan como resultado una definición más acorde a la realidad que se trata de entender y eventualmente modificar.

Pasar de una noción sencilla e intuitiva a un constructo complejo y explicativo de un fenómeno social conlleva, por parte de quien lo realiza, entender y proponer formas de extraer de la realidad un conjunto de elementos que son ordenados desde determinados referentes e intencionalidades, haciendo posible con esto aproximarse a una comprensión de la manera en que se dan los mecanismos sociales a partir de los sujetos, sus interacciones y las estructuras en las que se encuentran inmersos (Elster, 2005), lo cual incluye sus posiciones políticas, intelectuales e ideológicas y, en el caso de instituciones como el INEE habría que añadir, desde las culturas de las profesiones de quienes intervienen.

Sin embargo, lo que se quiere destacar en este proceso de construcción es el uso del método científico como la puerta de entrada que hace posible construir y acceder al fenómeno social — por ejemplo, el currículo-, ya sea para fines de descripción, comprensión, explicación, e incluso para intervenir en él. En ese sentido, la cientificidad, entendida como la inteligencia y racionalidad de las ciencias sociales (Díaz, 2005), se basa en la capacidad de construir un discurso articulado, referenciado y consistente, que se respalda en el uso de métodos susceptibles de ser replicados, y en esa medida validados, los cuales se dinamizan a partir de la actividad investigativa y en la participación de los productos que resulten de la aplicación de esta racionalidad, tengan dentro de las diferentes comunidades epistémicas de los campos del saber.

Es entonces que en el método científico social, conceptos como rigor, verificabilidad, comunicabilidad, aplicabilidad y plausibilidad (Kuhn, 2013; Habermas, 1987) cobran valor y, como veremos, son retomados por la actividad evaluativa para determinar las características de sus propias acciones. Aunque antes habrá que entender que en esta construcción de la perspectiva científica, tan importante es el método como el objeto de investigación.

El objeto se refiere a la forma en que es parcelado el fenómeno social, dando pie a una aproximación analítica que se traduce en la acción investigativa (García, 2001), es decir, que al articularse el objeto con la acción, la investigación entendida como actividad puntual que toma el método científico para sus propios fines, se moviliza a favor de procesos que se dan de manera continua y que se van re-ajustando, re-armando y re-creando a partir de las determinaciones de quienes lo investigan, en tanto el objeto de investigación no existe de manera independiente a la actividad del investigador y a su forma de abordarlo, lo cual incluye los supuestos teóricos, creencias y valores de los que este parte (Jiménez, 2012; Becher, 2009). 
La investigación, por tanto, no es una actividad neutral sino que entra en relación con el momento, los sujetos, el tipo de preguntas que construye y busca resolver a favor de los propósitos, tanto de la propia investigación pero, también, de las mismas finalidades de la institución o coyuntura en cuestión. Tomando en consideración lo anterior, la incorporación del currículo en el INEE, primero como objeto de investigación y después como objeto de evaluación, ha pasado por una serie de decisiones que en el terreno de la racionalidad científica tienen que ver con la construcción conceptual, el tipo de análisis que se hace y la división del objeto que se establezca en función de las condiciones institucionales, políticas y axiológicas en las que nos encontramos.

Este proceso incluye la incorporación, explícita e implícita, de marcos legales, éticos, morales y políticos desde los cuales se concibe el objeto y que determinan los propósitos a alcanzar, así como el tipo de valoraciones que en el contexto del INEE se pretendía llevar a cabo como parte de su misión evaluadora, que si bien está dada por mandato constitucional, requería para su legitimidad - fundarse en una racionalidad científica que le permitiera cumplir con características de rigor, verificación, comunicación de resultados, comprobación, uso y su eventual transformación en función de un paradigma de producción del conocimiento.

Por tanto, hablar de investigación y evaluación no significa abordar dos mundos separados en tanto diferentes y en los que se tenga que desistir de uno para lograr el otro; la evaluación en sí misma, para lograr sus fines de emisión de juicios de valor más allá de cumplir con fines exclusivamente normativos, ha de tomar los planteamientos de la ciencia y la investigación para atender las necesidades sociales de la actividad que realizamos desde la producción de conocimiento.

Considerando lo arriba expuesto, es importante reconocer que el proceso de investigación para la construcción del objeto de evaluación, el cual incluye el campo del currículo, tiene una historia en la que participa una comunidad y que presenta puntos en común y perspectivas encontradas, así como tensiones fuertes entre quienes diseñan el currículo y quienes lo implementan y, en ese sentido, el tipo de conversaciones que generan al respecto (Perrenoud, 2011; Gimeno, 2007; Pinar, 2003).

Todas estas discusiones sostenidas en el interior de la DECME, así como las que se dan con la comunidad epistémica del currículo y en la revisión de literatura, junto con los resultados obtenidos en el proceso de construcción de las evaluaciones, se encuentran plasmadas en el marco referencial y metodológico (INEE, 2019), el cual, antes de publicarse como un documento público, fue una guía para plantear líneas de investigación-acción que dan cuenta del propósito que se tenía como área dentro del instituto.

A continuación se resaltan algunos puntos contenidos en este documento, sobre todo con la finalidad de situar el currículo como un elemento central en el proceso educativo, y por tanto en el de la evaluación; y por otro lado, para ilustrar por qué se dice que no se puede evaluar sin investigar, así como no se puede investigar sin innovar.

Diálo@os

sobre Educación año 11 | número 20 | enero-junio 2020 | ISSN 2007-2171 
El análisis del currículo, como eje y mediador que estructura la práctica educativa producida en la interacción de alumnos y docentes, y que desde su implementación condiciona el conjunto de las políticas educativas, se basa en una comprensión de los para qué, los qué, los por qué y los cómo (INEE, 2019); en la respuesta de estas preguntas es donde se pueden identificar los componentes de la acción educativa, así como discutir las posiciones de los actores que intervienen de manera directa e indirecta en ella, y — para el caso de la DECME— dando una definición teórica, epistemológica y metodológica pero también práctica, del currículo como objeto de investigación, entendido como:

referente y organizador de las finalidades educativas en el ámbito formal y también de lo que realmente sucede en el mismo; es articulador de la vida escolar y núcleo en el funcionamiento de esta; configura la práctica educativa y motiva la actividad diaria del aula (INEE, 2019: 13).

Al evocar esta definición nos encontramos con que las variables del currículo no son externalidades o factores concretos que puedan observarse de manera independiente del sujeto, sino que se despliegan en la relación que los sujetos establecen entre ellos (Dussell, 2014), y abarca sus creencias, conceptos previos, prácticas y juicios que intervienen en la lectura, apropiación y representación que hacen de los elementos con los que apoyan u orientan su práctica de enseñanza y los procesos de aprendizaje.

Lo anterior implica un abordaje complejo, que a la vez sea capaz de identificar y analizar las "concreciones curriculares" en las que el currículo se transforma o traduce. En ese sentido, y siguiendo a De Ibarrola (2012; 2006), el currículo no se reduce al plan o programa de estudios, sino que se manifiesta en muchas facetas de las prácticas que ocurren en el aula. Por ello, el abordaje que se pretende distingue diferentes dimensiones de lo curricular que se abordarán más adelante, conceptualmente ancladas en un planteamiento de la evaluación del currículo como un proceso que De Alba (1991) y Díaz Barriga (1995) definen como el resultado de la reflexión, el análisis crítico y la síntesis valorativa, para lo cual se requiere generar propuestas que den cuenta de estas imbricaciones y posiciones de los actores que intervienen en su definición y puesta en marcha, que lo transforman y negocian en sus actos (Perrenoud, 2011; Gimeno, 2007).

\section{El camino andado}

El proceso investigativo detonado a partir de la evaluación del currículo, debido a la necesidad de hacer recortes metodológicos, ha propiciado el establecimiento de fronteras para hacer plausible el análisis curricular, evitando profundizar en un análisis filosófico que aún cuando es muy socorrido y válido en el grupo de investigación del currículo (García, 2014), es insuficiente cuando se pretende derivar propuestas de mejora concretas o identificar problemáticas que 
sean comprensibles, no solo para quienes somos parte de una comunidad epistémica sino para los potenciales usuarios y audiencias.

Respecto de la investigación sobre currículo que se ha hecho en México, Díaz Barriga (2013), en una edición del Consejo Mexicano de Investigación Educativa sobre los estados del conocimiento, señala que la investigación se caracteriza por una amplia diversidad de enfoques, entre los que se destacan aquellos que atienden una perspectiva poscrítica y posestructuralista del currículo y sus manifestaciones concretas; otro, de corte genealógico o foucaltiano, interesado en el análisis de los conceptos que se manejan en el discurso curricular; un tercero, más centrado en la acción curricular o la nueva didáctica; y por último, un enfoque con una visión más interesada en conocer y documentar experiencias concretas en diferentes campos del saber, sobre todo en el nivel universitario.

Frente a esta pluralidad de aproximaciones y con el fin de atender el sentido práctico que tiene el trabajo en una institución de gobierno, la forma en que se ha resuelto esta situación dentro del equipo de la DECME ha sido mediante la identificación de tres dimensiones: diseño, implementación e impacto, y en las que se pueden enmarcar los procesos, actores, recursos e interacciones que abarcan el currículo, y que son consistentes con la investigación realizada en el campo del currículo (Stabback, 2016), permitiendo con esto avanzar en la configuración de las evaluaciones.

La dimensión del currículo diseñado parte de una comprensión de este como prescriptor de la práctica didáctico-pedagógica, como documento político-normativo y guía de la misión educativa del Estado, en la cual se identifican y ordenan los objetivos de aprendizaje y los contenidos, se determinan las trayectorias y los niveles, sus enfoques, los materiales y recursos que se derivan, y las secuenciaciones que se proponen y prescriben desde una posición externa a la práctica educativa (INEE, 2019).

En cuanto a la dimensión de implementación, esta se refiere a la forma en que los actores en la escuela retoman el currículo diseñado y orientan su práctica, misma que es influida por los perfiles profesionales de los docentes, sus creencias, expectativas y valores; el tipo de relaciones que establecen en el aula; las características sociales y culturales de docentes y alumnos; la historia y tradición pedagógica de determinado subsistema, y la forma en que estos elementos integran, interpelan, fortalecen o debilitan los procesos de enseñanza y aprendizaje de docentes y alumnos (Stabback, 2016).

La dimensión de impacto se asocia a los resultados que como proceso educativo se tienen principalmente en los alumnos, aunque no sólo vistos en función del aprendizaje sino en el cumplimiento de los objetivos que el propio currículo persigue, referidos a los beneficios para la vida personal y social de los estudiantes (Muñoz, 2003; Daviet, 2016); esta definición, si bien resulta lógica en términos de los procesos del currículo desde las tres dimensiones, se enfrenta a varios retos que más adelante se tratará de apuntar con mayor precisión.

DiáloQos sobre Educación año 11 | número 20 | enero-junio 2020 | ISSN 2007-2171 
La definición de estas dimensiones, como resultados del proceso analítico que recoge una idea científica de aproximación al objeto de estudio, a su vez ha hecho posible perfilar el tipo de evaluación que habrá de llevarse a cabo para enfrentar la tarea encomendada al INEE, en relación con el dar cuenta de todos los componentes, procesos y resultados que intervienen en el SEN. A la vez, ha constituido una solución práctica que desde el trabajo de investigación y la parcelación del objeto de evaluación permite hacer un uso racional de los recursos disponibles y generar información útil que consideramos está en el centro del proceso educativo, aportando elementos de información desde perspectivas poco abordadas por las diferentes áreas del instituto, relacionadas con la naturaleza didáctico-pedagógica de los procesos educativos.

El resultado de este trabajo derivó en un esquema que permite avanzar en dos sentidos: dar cuenta de las dimensiones del currículo y distinguir lo que metodológicamente era pertinente para cada dimensión. Así, como parte de dicho esquema, se consideró dar cuenta de la dimensión del diseño curricular, de la implementación curricular y del impacto curricular como objetos de evaluación. En el siguiente cuadro, tomado del documento referencial y metodológico antes mencionado, es posible identificar el qué, el cómo y el para qué, que se ponen en juego en cada dimensión y que han hecho las veces de guía para orientar el plan de evaluación que precede a cada uno de los trabajos llevados a cabo. 
Cuadro 1. Qué, para qué y cómo evaluar el currículo

\begin{tabular}{|c|c|c|c|}
\hline $\begin{array}{l}\text { Dimensiones del CU- } \\
\text { rrículo }\end{array}$ & $\begin{array}{l}\text { ¿Qué evaluar del cu- } \\
\text { rrículo? }\end{array}$ & $\begin{array}{l}\text { ¿Para qué evaluar el } \\
\text { currículo? }\end{array}$ & $\begin{array}{l}\text { ¿Cómo evaluar el cu- } \\
\text { rrículo? (ejemplos) }\end{array}$ \\
\hline Diseño & $\begin{array}{l}\text { Objetos y referentes } \\
\text { curriculares }{ }^{*} \text { de ma- } \\
\text { nera independiente o } \\
\text { agrupada. }\end{array}$ & $\begin{array}{l}\text { Para valorar el diseño } \\
\text { curricular, en tanto } \\
\text { prescribe desde lo } \\
\text { normativo, el trabajo } \\
\text { de la escuela y el aula. }\end{array}$ & $\begin{array}{l}\text { Estudios de caso, } \\
\text { grupos de enfoque, } \\
\text { análisis documental, } \\
\text { jueceo, rúbricas, en- } \\
\text { trevistas, cuestiona- } \\
\text { rios. }\end{array}$ \\
\hline Implementación & $\begin{array}{l}\text { Condicionantes insti- } \\
\text { tucionales y escolares; } \\
\text { disposiciones, pensa- } \\
\text { miento y experiencia } \\
\text { docentes, e interaccio- } \\
\text { nes de los sujetos que, } \\
\text { de manera relacionada, } \\
\text { toman lugar en el espa- } \\
\text { cio escolarizado. }\end{array}$ & $\begin{array}{l}\text { Para valorar la relación } \\
\text { directa e indirecta que } \\
\text { se establece entre la } \\
\text { práctica educativa de } \\
\text { los sujetos (alumnos, } \\
\text { docentes y directivos) } \\
\text { con el currículo pres- } \\
\text { crito. } \\
\text { O bien para valorar la } \\
\text { calidad de las condi- } \\
\text { ciones institucionales } \\
\text { o personales y su in- } \\
\text { fluencia en la práctica } \\
\text { educativa. }\end{array}$ & $\begin{array}{l}\text { Cuasi-experimentos, } \\
\text { estudios de caso, gru- } \\
\text { pos de enfoque, obser- } \\
\text { vación directa, entre- } \\
\text { vistas, cuestionarios, } \\
\text { instrumentos estanda- } \\
\text { rizados. }\end{array}$ \\
\hline Impacto & $\begin{array}{l}\text { Logro educativo, tra- } \\
\text { yectorias educativas y } \\
\text { externalidades. }\end{array}$ & $\begin{array}{l}\text { Para valorar los efectos } \\
\text { que tiene el currículo en } \\
\text { la formación del sujeto } \\
\text { individual y como ente } \\
\text { social, y en la sociedad } \\
\text { en general. }\end{array}$ & $\begin{array}{l}\text { Pruebas objetivas de } \\
\text { rendimiento académico, } \\
\text { análisis transversales de } \\
\text { trayectorias, estudios } \\
\text { de empleabilidad. }\end{array}$ \\
\hline
\end{tabular}

Fuente: INEE (2019).

A continuación, se presentan los avances logrados en productos concretos de cada dimensión del currículo salvo la del impacto, ya que ésta implicaba un trabajo que organizacionalmente superaba a la DECME; sin embargo, se ha querido visibilizarla para que en un futuro se pueda seguir avanzando.

Para el caso del diseño del currículo, se recurrió a metodologías, sobre todo de pequeña escala y cualitativas, que hicieran uso del análisis documental y de los discursos plasmados en los diferentes objetos curriculares que prescribe el currículo. Por ello, es importante partir de

\footnotetext{
* De acuerdo con el documento de "Orientaciones técnicas para la evaluación del diseño curricular. Evaluación de contenidos y métodos educativos" (INEE, 2019), se entiende por objetos y referentes curriculares a los documentos oficiales o normativos que orientan el trabajo escolar y que tienen como referentes las política educativas que determinan su enfoque, naturaleza y alcances.
}

\section{Diálo Dos}


la idea de que una evaluación del diseño curricular no es lo mismo que elaborar un estado del arte. Para cada evaluación se decidió si se focalizaba un área disciplinar del currículo o si se valoraba algún elemento transversal a cualquier espacio curricular, y se definieron las categorías o ejes que servirían como criterios para hacer la valoración (INEE, 2019). Dichas evaluaciones, por mandato legal, correspondían al tramo de la educación obligatoria; para el caso de México, hasta el 15 de mayo de 2019 abarcaba, de acuerdo con la Clasificación internacional normalizada de la educación de la UNESCO (2011), los niveles del 0 al 3. En el caso de las evaluaciones de un área disciplinar se sumó la perspectiva comparada, la cual pretende ofrecer marcos de referencia para mirar el currículo mexicano, en donde se identifican similitudes y diferencias entre currículos y tendencias formativas en el área disciplinar en cuestión. Las evaluaciones de diseño curricular que se hicieron o coordinaron en la DECME son las siguientes:

- Estudio comparativo de la propuesta curricular de matemáticas. Es una evaluación que estuvo a cargo de Teresa Rojano, del Departamento de Matemática Educativa del Centro de Investigación y Estudios Avanzados (Cinvestav) del Instituto Politécnico Nacional, sobre la calidad del diseño curricular vigente en 2016 en el área de Matemáticas de todos los niveles de la educación obligatoria en México. Se compara el currículo mexicano con el de tres países (Corea del Sur, Chile e Inglaterra). Para ello se consideran, por un lado, criterios de calidad del diseño curricular de acuerdo con las dimensiones de relevancia y pertinencia señaladas en la ley del INEE, así como con las dimensiones de congruencia y consistencia internas; por otro, para la comparación con las propuestas curriculares de otros países, considera elementos del diseño curricular, como el marco contextual, los fundamentos, las intenciones educativas, los contenidos escolares, el enfoque pedagógico y el modelo de evaluación del aprendizaje (Rojano y Solares, 2017). Se encuentra disponible en el sitio de internet: https://www.inee.edu.mx/wp-content/ uploads/2019/01/P1F210.pdf

- Estudio comparativo de la propuesta curricular de ciencias. Es una evaluación en la que colaboraron integrantes de la DECME y José Antonio Chamizo, de la Facultad de Química de la Universidad Nacional Autónoma de México (FQ-UNAM), sobre la calidad del diseño curricular vigente en 2016 en el área de Ciencias de todos los niveles de la educación obligatoria en México. Se analizan los planes y programas de estudio de cinco países (Brasil, Chile, Corea del Sur, Holanda y el Proyecto 2061, desarrollado en Estados Unidos) con la intención de caracterizar la propuesta curricular de cada uno, para identificar similitudes y diferencias en sus planteamientos y compararlos con la propuesta mexicana. Los referentes son los criterios de calidad educativa y el derecho a la educación. Este texto ofrece una oportunidad para reflexionar sobre la congruencia, consistencia y pertinencia del currículo de ciencias para la educación obligatoria en México (Reynoso y Chamizo, 2017). Se encuentra disponible en el sitio de internet: https://www.inee.edu.mx/wp-content/uploads/2019/01/P1F211.pdf 
- Estudio de la política curricular para población indígena. Es una evaluación que estuvo a cargo de Ana Laura Gallardo del IISUE-UNAM, que exploró la política curricular vigente en educación básica para la atención a población indígena, mediante la revisión documental de la normatividad, documentos curriculares y la Consulta previa, libre e informada a pueblos indígenas, realizada por el instituto; la realización de entrevistas a funcionarios responsables de la política; y visitas a escuelas para valorar la puesta en marcha de la política curricular. Este estudio sirvió como insumo en la elaboración de las Directrices para mejorar la atención educativa a niñas, niños y adolescentes indígenas, publicadas por el Instituto, las cuales se encuentran disponibles en la liga: https://www.inee.edu.mx/wp-content/ uploads/2018/12/P1F104.pdf

- Evaluación del diseño curricular para la formación ciudadana en la educación obligatoria. Este trabajo se enmarca en el cambio del currículo oficial de la educación obligatoria en el país; el objetivo de esta evaluación es dar cuenta de la forma en que se incorpora el enfoque de formación ciudadana en el nuevo currículo; para ello, las encargadas de la investigación, Sylvia Conde y María Eugenia Luna, de la asociación civil Colectivo Albanta, y Benilde García, de la Facultad de Psicología de la UNAM, identifican cuatro dimensiones de análisis en la construcción del sujeto como entidad moral, política, social y con dignidad y derechos (INEE, 2019). Estas categorías de análisis, a su vez se entrelazan con los atributos del diseño curricular elaborado por la propia DECME, permitiendo con ello construir un marco analítico consistente entre las diferentes evaluaciones y estudios que abordan la dimensión del diseño. Se encuentra disponible en el sitio de Internet: https://www.inee.edu.mx/wp-content/uploads/2019/08/P1F232.pdf

- Análisis del diseño del componente de autonomía curricular. Plan y programas de estudio 2017. En este estudio se analiza el nuevo componente del plan de estudios llamado autonomía curricular, del plan de estudios 2017, el cual consiste en que las escuelas deciden sobre una franja horaria de la jornada de cada escuela y los contenidos, enfoques y organización del espacio, lo cual contrasta con la tradición mexicana de tener un currículo altamente centralizado y prescriptivo. A diferencia de los otros estudios, este se desarrolló de manera interna en la Dirección por los autores de este artículo, y el objetivo es generar recomendaciones acerca del diseño con el propósito de hacer los ajustes necesarios para su implementación. Analíticamente, el estudio revisa el planteamiento curricular de este componente desde cuatro ejes: inclusión, flexibilidad, gestión y liderazgo, y adiciona los atributos del diseño curricular definidos de manera previa en el documento de criterios, elaborado por la dirección (INEE, 2019). Se encuentra disponible en el sitio de Internet: https://www.inee.edu.mx/wp-content/uploads/2019/05/ P1F231.pdf

Sobre la dimensión de implementación curricular, se han desarrollado evaluaciones tanto de gran escala como de mediana y pequeña, también se ha recurrido al enfoque cualitativo en el caso de las realizadas a mediana y pequeña escala. En esta dimensión, dada su naturaleza, se 
ha optado por focalizarla en algún nivel educativo para dar cuenta de los elementos, categorías o criterios de interés; en todos los casos, son dos los referentes para hacer la evaluación: el currículo vigente para dicho nivel educativo y el cumplimiento del derecho a la educación. Así se busca dar cuenta de la forma en que el currículo implementado promueve, permite o dificulta la atención de los derechos en la educación, los cuales tienen que ver con que la educación que se ofrezca sea adaptable y aceptable.

Las evaluaciones de implementación curricular realizadas por la DECME son las siguientes:

- Evaluación de la Implementación Curricular en Educación Media Superior (EIC-EMS), con la que se buscó dar cuenta de la implementación del Marco Curricular Común (MCC) propuesto para dicho nivel educativo en 2008, a partir de recuperar las opiniones y valoraciones de actores educativos (estudiantes, docentes y directivos) de ocho tipos de planteles distintos, acerca de las acciones de formación y profesionalización que se emprendieron para la implementación del MCC, las prácticas de aula preponderantes, las acciones de gestión emprendidas para apoyar al MCC y las valoraciones de planes y programas de estudio (INEE, 2018). De esta evaluación se desprendieron dos productos: un informe de resultados generales y uno complementario que se enfocó en el tema del abandono escolar, visto desde la implementación del currículo. Ambos se encuentran disponibles en las siguientes ligas de Internet: https:// www.inee.edu.mx/wp-content/uploads/2018/12/P1D248.pdf; https://www.inee.edu.mx/wp-content/ uploads/2019/08/P1D256.pdf

- Evaluación de la Implementación Curricular en educación preescolar (EIC-Pree), con la que se buscó dar cuenta de las creencias que educadoras y líderes para la educación comunitaria tienen respecto al currículo, la enseñanza y el aprendizaje. Con este estudio se pretende tener una aproximación al pensamiento de los docentes como un elemento de la implementación curricular que permite comprender que cuando un currículo se implementa ya ha pasado por la mediación del docente, conformada por sus experiencias, conocimientos, ideas y creencias; esto puede ofrecer información para retroalimentar el diseño curricular y elementos para comprender la implementación en este nivel educativo (INEE, 2019). En este estudio participan preescolares generales, indígenas y privados, además de preescolares comunitarios del CONAFE. Se encuentra disponible en el sitio de internet: https://www.inee.edu.mx/wpcontent/uploads/2019/05/ResultadosElC-Preescolar2017.pdf

Por otra parte, las evaluaciones de esta dimensión a mediana y pequeña escala se han concentrado en el nivel de educación media superior, específicamente en tres tipos de servicio: los Centros de Educación Media Superior a Distancia (EMSAD), los Telebachilleratos Estatales (TBE) y los Telebachilleratos Comunitarios (TBC), estos últimos representan una de las estrategias de la administración federal que concluyó en 2018, para la ampliación de la cobertura en este nivel educativo. Estas evaluaciones han sido de corte cualitativo y son las siguientes: 
- Modelo de Telebachillerato Comunitario y su operación en los estados. Este estudio estuvo a cargo de Eduardo Weiss, del DIE-Cinvestav, quien junto con un equipo de investigadores exploró las estrategias para la operación de estos servicios. Especialmente para el TBC, identificaron que su diseño curricular encontraba tensiones al momento de ser operado, que era un servicio altamente valorado por los estudiantes y las comunidades, pero que estaba lleno de inequidades para los docentes (Weiss, 2017). Este estudio se encuentra disponible en el sitio de Internet: https://www.inee.edu.mx/wp-content/ uploads/2018/12/P1C155.pdf

- Avances y dificultades en la implementación del Marco Curricular Común Telebachillerato estatal, Educación Media Superior a Distancia y Telebachillerato comunitario. Este estudio estuvo a cargo de Carlota Guzmán, del CRIM-UNAM, quien junto con un equipo de investigadores exploró la implementación del Marco Curricular Común en estos tipos de servicios en cinco entidades federativas. Este estudio busca complementar la información obtenida con la evaluación a gran escala en el mismo nivel educativo, toda vez que en dicha muestra no se consideraron estos servicios educativos. Así, en este estudio se exploran las acciones de formación y profesionalización para docentes y directivos, las prácticas de aula preponderantes, las acciones de gestión escolar impulsadas como apoyo al MCC, y se recuperan las valoraciones que estudiantes, docentes y directores tienen de los planes y programas de estudio (Guzmán, 2018). Este estudio se encuentra disponible en el sitio de Internet: https://www.inee.edu.mx/ wp-content/uploads/2018/12/P1C234.pdf

\section{Orientaciones técnicas para la evaluación del diseño curricular}

Además de los estudios y evaluaciones mencionadas, se hicieron esfuerzos por impulsar evaluaciones externas e internas de currículo; así se generaron orientaciones para las evaluaciones de diseño curricular (INEE, 2019) que buscan contribuir a su elaboración, reconociendo que no solo el instituto concentraba estos ejercicios, sino que ocurrían en otros espacios e instancias. Las orientaciones buscaron ofrecer herramientas para valorar las diferentes propuestas de enseñanza que desarrollan. Dichas orientaciones permiten construir esquemas ad hoc de evaluación-investigación en los que la innovación para concebir, diseñar y analizar la información con fines de evaluación ha sido esencial, en la medida de que la naturaleza de nuestro objeto de estudio se manifiesta de manera amplia y compleja.

Como parte de este documento se definió un conjunto de atributos, ya mencionados arriba, cuya finalidad es identificar rasgos mínimos a partir de los cuales se pueda hacer la evaluación; estos atributos se presentan de manera resumida en el siguiente esquema:

Diólopos sobre Educación 
Figura 1. Atributos del diseño curricular

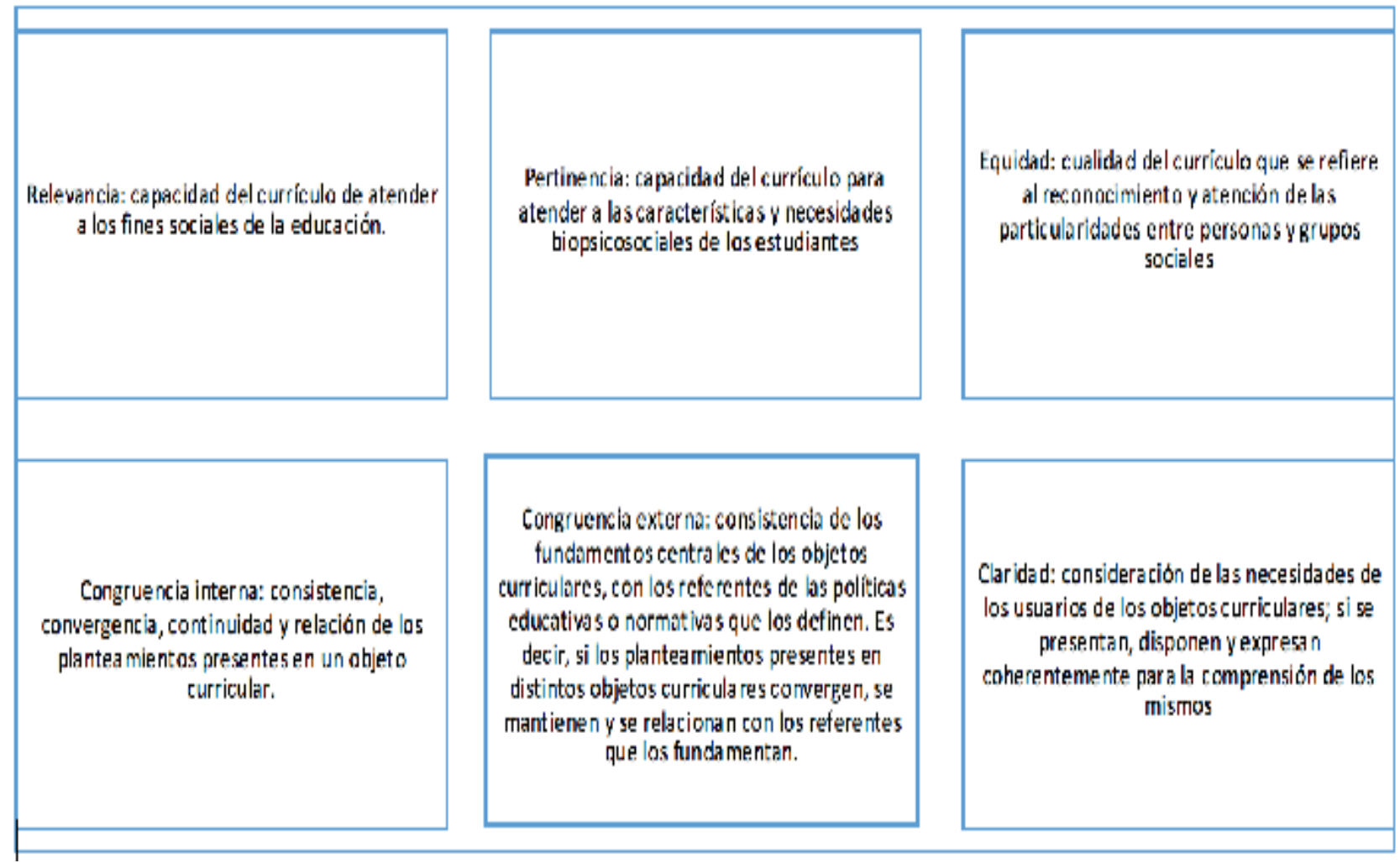

Fuente: INEE (2019).

El trabajo hasta aquí avanzado ha sido resultado de esta construcción teórica y metodológica del objeto de estudio, el cual tiene las características de no ser estático ni unívoco; de ahí la importancia de construir una definición conceptual, pero también operativa, no solo acerca de cómo entender el currículo, sino además, cómo indagar en él con fines evaluativos. En el siguiente apartado se abordarán algunos retos de índole metodológica y organizacional, que han supuesto el dar cabida a un área de trabajo dentro del extinto Instituto Nacional de la Evaluación en México y cómo esta visión acerca de la evaluación se contrapone o articula a otras visiones acerca de la tarea evaluativa desde concepciones distintas a la pedagogía.

\section{Avances y retos en la evaluación del currículo}

Ante el camino andado, es preciso tener claridad sobre los principales avances y retos que en el diseño de las evaluaciones hemos registrado, y que toca puntos como las limitaciones en el uso de las perspectivas cuantitativas y cualitativas, y las tensiones organizativas que en el interior del instituto seccionan elementos del currículo, limitando con esto el propio valor del objeto de evaluación en su conjunto a favor de sus partes; mientras que en el exterior no existe claridad en torno a la responsabilidad de los actores que participan de la evaluación del currículo, crean- 
do con esto diferentes niveles de retos que condicionan la realización de las evaluaciones del currículo como fue conceptualizado.

\section{Avances y retos en la formulación metodológica}

En cuanto a la construcción metodológica y la consideración que demanda el trabajo con enfoques cualitativos y cuantitativos, e incluso mixtos - como el caso de la evaluación sobre autonomía curricular-, se advierte la necesidad de generar evidencia que sustente los juicios que se pueden construir como parte de la evaluación, a partir de un diseño metodológico adecuado que recupere las bondades de ambos enfoques, y a la vez justifique y haga viable la encomienda del instituto de generar evaluaciones de escala nacional que den cuenta de los componentes, procesos y resultados del sistema educativo y del currículo para nuestro caso.

En este sentido, los enfoques de investigación se colocan como marcos directivos que delimitan los alcances de nuestro trabajo; así, la metodología cuantitativa como primer acercamiento a un fenómeno, permite identificar la presencia y distribución de una situación específica en una población determinada. Entonces su contribución consistiría en conocer de qué manera una decisión, una política, un programa implementado o una interacción dada, afectan en términos generales la dinámica individual y social de las personas. Mientras que el uso de metodologías cualitativas nos permite acceder a un nivel más profundo de la subjetividad de las personas con la finalidad de conocer las formas de cómo vivencian, resignifican y dotan de sentido la actuación de las personas, así como el tipo de relaciones que establecen en su cotidianidad, entre ellos o entre ellos y las organizaciones en las que participan y los discursos que en torno a la educación se construyen, entendiendo que el currículo es en sí mismo un discurso (De Alba, 1991).

Sin embargo, el estudio de los fenómenos sociales, cuando menos desde las tradiciones contemporáneas de las ciencias sociales, se ha presentado como una disyunción lógica entre el conocer los sistemas y procesos o a los sujetos en su contingencia (Galindo, 2012). Frente a este dilema, los modelos de conocimiento y sus técnicas se han visto atrapadas entre la posición encontrada de lo cuantitativo versus lo cualitativo. Esta situación ha conducido a confundir el enfoque epistemológico con la selección de técnicas (Schmelkes, 2001). La tensión entre lo cuali y lo cuanti revela por tanto un problema mayor, la comprensión de los fenómenos desde dos perspectivas: lo macro frente a lo micro.

En oposición, se puede señalar que la articulación de las metodologías que son propias de los enfoques macroscópicos y microscópicos no son inconmensurables a partir de sus elementos epistémicos sino, en todo caso, desde la posición del investigador que lo hace ver así. Por otra parte, la educación como objeto múltiple de estudio no responde a una visión disciplinar única pero sí temática, en donde las formas de abordar el objeto de conocimiento se han

Diólo@os sobre Educación año 11 | número 20 | enero-junio 2020 | ISSN 2007-2171 
mostrado como variadas y de acuerdo a las características de la comunidad de investigación y disciplina que la estudia (Weiss y Gutiérrez, 2009).

Sobre las limitantes en el uso de enfoques cuanti/cuali, el racionalismo crítico (Hernández, Romero, Bracho, 2005) como principio del conocimiento inferencial y probabilístico, se constituye en un elemento fuerte al momento de abordar la realidad construida socialmente; la metodología cuantitativa en el ejercicio de la comprobación/refutación permite generalizaciones que son válidas en la medida que hace uso de estructuras lógicas capaces de darle respuestas puntuales a una pregunta basada en una medición que describa o haga posible la predictibilidad en el comportamiento del fenómeno estudiado (Hernández, Fernández y Bautista, 2010), aunque es importante apuntar que existe un reduccionismo propio de los enfoques racionalistas al presentarse como estructurantes únicos de la realidad (Massé, 2003). Frente a estos sesgos, es vital tener claro que su presencia se da a partir de los recortes metodológicos hechos por el propio investigador-evaluador y no por la perspectiva epistemológica o metodológica per se.

Por otra parte, a pesar de que un modelo de investigación de tipo hermenéutico-interpretativo nos permitiría profundizar en aspectos particulares de los sujetos, su visibilidad como primer paso, previo a la profundización de fenómenos concretos, parece depender de la producción de datos que nos permitan ubicar las características de los contextos, prácticas y representaciones que determinan e interaccionan con la experiencia vital de los sujetos, con el propósito de dotarlos de iconicidad.

A partir de lo hasta aquí apuntado, se puede concluir que el uso del enfoque cuantitativo, para el caso de la evaluación del currículo, ha permitido realizar amplios mapeos que aportan información para aproximarse, y en esa medida comprender las prácticas de los docentes respecto al currículo y cómo estas son condicionadas por el diseño y situación institucional en que se encuentran las escuelas, los docentes y los estudiantes. Las evaluaciones de tipo cualitativo han dado pie a comprender las dinámicas que en el nivel micro se establecen entre actores, especialmente en el caso de los tipos de servicios cuya representatividad es exigua y los que, como en el caso de los Telebachilleratos (TB), constituyen ofertas con características muy específicas, así como un diseño institucional muy reciente que coincidentemente es el que atiende a poblaciones con mayor desventaja, a la par que nos ha permitido acceder al nivel del discurso del currículo prescrito.

Con base en lo anterior, podemos afirmar que el conocimiento para la producción del currículo, sea con fines de investigación o evaluación, requiere la complementariedad de los métodos en tanto este no se agota en una sola visión metodológica ni corriente epistemológica, así como en una precisión en torno a qué se quiere y se puede evaluar en función de nuestra posición dentro del entramado institucional del que formamos parte y en atención a nuestros marcos de referencia que responden a la pedagogía y a la didáctica, y que derivan en constructos a evaluar. 
Así, el estudio de un tema casi exclusivo del currículo — como son los procesos de enseñanza-aprendizaje - requiere, previo al diseño metodológico, posicionarse teóricamente sobre su concepción; lo cual se aleja, por ejemplo, de las evaluaciones de políticas que dan por hecho la existencia de estos procesos, sin necesariamente profundizar en ellos porque el foco está puesto en otras variables. El interés de la evaluación del currículo está puesto, citando a Camilloni (2007: 22), en su dimensión didáctica, la cual aborda "la acción pedagógica, es decir, las prácticas de enseñanza [y según los autores de este artículo, abordando con esto el aprendizaje] y que tiene como misión describirlas, explicarlas y fundamentar y enunciar normas para la mejor resolución de los problemas que estas prácticas plantean a los profesores".

En función de lo arriba citado, reconocemos que la misma formulación e investigación en torno al currículo implica un proceso de diseño y evaluación en los que nos hemos basado; considerando esto, es importante quitarnos la idea de que no hay evaluación del currículo en México (Díaz-Barriga, 1995) puesto que esta sí existe; sin embargo, en su mayoría responden más a la valoración que se puede realizar en el marco de la actividad académica, que por lo regular son esfuerzos de corta escala que focalizan aspectos particulares, poblacional e institucionalmente limitados.

De ahí que el campo de la evaluación curricular a gran escala, que no se propone medir resultados o logros de aprendizaje — como son las pruebas de logro nacionales o internacionales-, sino la interacción didáctica de los sujetos, es un espacio de muy reciente construcción; el INEE es el primer gran espacio donde se están realizando este tipo de evaluaciones de currículo, pero que a mediano plazo abrirá la posibilidad de contar con líneas base e información que, como ya se dijo, permitan aproximarse a la complejidad de lo que implican las relaciones didácticas de los sujetos escolares para ver cómo se transforman, que recolecten cada vez más datos que nos guíen acerca de las variables que realmente inciden en el aprendizaje, y a su vez alimenten el amplio campo de la investigación y evaluación curricular, tanto del tipo cuantitativo como cualitativo.

De esta manera, el reto está en traducir todas estas condicionantes y consideraciones de la construcción metodológica de la investigación y producción de conocimiento en modelos de evaluación ad hoc a las características, los constructos y los procesos que se busca indagar en cada una de las tres dimensiones de la evaluación. Así como innovar conceptual y metodológicamente en el diseño de evaluaciones de impacto que no caigan en la valoración de productos concretos o resultados vistos como objetos, sino desde marcos distintos de valoración que permitan apreciar la contribución que el currículo tiene en la formación de las personas.

Por otra parte, la construcción metodológica de la evaluación no solo pasa por elegir los enfoques; debe considerar la disponibilidad de recursos en función de una ponderación de costos-beneficios, que en estricto sentido rompería con el cuadro axiológico que orienta la investigación, pero que no es posible dejar a un lado. Es así que valores propios del diseño de pro-

Diálo@os

sobre Educación

TEMAS ACTUALES EN INVESTIGACION : año 11 | número 20 | enero-junio 2020 | ISSN 2007-2171

16 
gramas y políticas públicas como es el uso racional de recursos en función de la maximización de los beneficios, la eficacia, eficiencia, transparencia y rendición de cuentas, son incorporadas al diseño mismo de las evaluaciones, generando con esto aparentes tensiones con un enfoque puramente investigativo, que nos lleva al siguiente punto: el que refiere al entramado organizativo en el que se realizan nuestras evaluaciones.

\section{Avances y retos de la evaluación del currículo en el entramado organizacional del INEE}

De acuerdo con Ramírez (2000), las organizaciones, entre las que se incluyen las burocráticas, están caracterizadas por sus grados máximos de estratificación, especialización y división del trabajo interno; lo cual deriva en la construcción de agendas de trabajo y procesos de planificación que como órgano de gobierno estamos obligados a realizar y que refleja los asuntos prioritarios para su atención, institucionalizándolos, regulándolos y normándolos.

Para el INEE, la reforma de 2013 supuso incrementar sus funciones y atribuciones que permitieran dar cuenta de la evaluación del conjunto del SEN, aunque poniendo especial énfasis en temas como la emisión de directrices, lineamientos y en regular los procesos relacionados con el Servicio Profesional Docente (SPD), así como en fortalecer el trabajo que se venía haciendo en la evaluación del logro de aprendizajes de los alumnos.

Este proceso de institucionalización, entendido como la formalización de una estructura o reglas que organizan la acción (De la Rosa, 2002), va respondiendo en función de la implementación de las que están en las mismas leyes y normatividades internas, a las demandas o necesidades que se van generando. Es así que el tema del currículo que abarca aspectos de organización escolar, trabajo docente, atención a estudiantes, valoración de procesos de enseñanza y aprendizaje, convivencia escolar e incorporación de enfoques de derechos y equidad, se colocaron en un primer diseño institucional como asuntos dislocados y abordados a partir de otros referentes con igual legitimidad, en tanto atienden las diferentes dimensiones de la educación como son la económica, política y social (De Ibarrola, 1985).

Al margen de esto, el reto actual está en entender la forma en que las diferentes perspectivas de abordaje con el que se trabajan las evaluaciones y el diseño de políticas en el interior del Instituto pueden confluir sin traslaparse, para dar cuenta de valoraciones más integrales en torno a los fenómenos educativos desde sus dimensiones económicas, políticas y sociales e introduciendo la dimensión curricular, es decir, la valoración didáctico-pedagógica del proceso de enseñanza y aprendizaje en particular, como un nuevo espacio de trabajo institucional que a la vez refleje la importancia que el tema tiene en el ecosistema de la investigación y evaluación de la educación fuera del INEE.

Para ello, será necesario generar nuevos focos de conocimientos y sensibilización en el interior del instituto —o de la ahora Comisión Nacional para la mejora Continua de la Educación- acerca de la importancia que tiene el currículo para evaluar y comprender la educación, 
y por qué es importante que la información obtenida con las evaluaciones del currículo sea utilizada y difundida por otras áreas como las de lineamientos, directrices o en el seguimiento a los Proyectos de Mediano Plazo (PEEME). Lo anterior, con el fin de dotar de mayor significatividad a su trabajo y a las audiencias a las que se dirige ya que, como lo señalan Stigler y Hiebert (2002), la clave para mejorar la calidad de la educación no sólo está en que se disponga de los recursos necesarios o en una focalización efectiva de acciones de política, sino en poner a disposición de los docentes recursos pedagógicos y didácticos efectivos para mejorar su práctica docente.

\section{Avances y retos en el contexto de la evaluación y lo normativo fuera del INEE}

En la investigación educativa la evaluación del currículo tiene un gran peso, en parte derivado de que el diseño y la implementación son procesos normados y una tarea exclusiva de las autoridades educativas, en los que la participación de otros actores como los académicos es marginal. Esta situación se da en todos los niveles del SEN, desde la educación básica hasta la superior. Para el caso del INEE, aun cuando estaba considerado en la ley que el instituto, como máxima autoridad evaluativa, tenía la potestad de evaluar todos los componentes del SEN —incluido el currículo-, existían lagunas jurídicas sobre su evaluación.

En el artículo 48 de la Ley General de Educación (DOF, 2013) se establecía que la Secretaría de Educación Pública (SEP) federal es la que de manera exclusiva determinará y evaluará el currículo de la educación básica, aunque pudiendo consultar a las Autoridades Educativas Locales (AEL), así como a otros actores en los que se encuentra el INEE. ${ }^{2}$ Esto, a pesar de los cambios a favor de la gobernanza del SEN y a que se observan —a partir del modelo educativo 2017- espacios de oportunidad y de mayor incidencia en el currículo, no sólo por parte del INEE sino de otros actores interesados que deben ser aprovechados.

No obstante, el artículo citado refleja la historia del currículo oficial y su evaluación, el cual supone un reto a las capacidades de los actores educativos quienes están acostumbrados a ver el currículo como algo ya dado, donde sólo los académicos pueden escudriñar y los docentes implementar; sin embargo, lo que se busca es que el currículo sea evaluado como parte de un proceso abierto en el que los diferentes actores participen, que además de servir para la contraloría social de los recursos, programas y políticas públicas, se instale en una lógica de constante revisión y fortalecimiento de la actividad didáctico-pedagógica que responda a necesidades y tiempos académicos, y no sólo a coyunturas políticas.

A pesar de que, como María de Ibarrola (2012) señala, en una perspectiva amplia de currículo se corre el riesgo de que todo pase por este, creemos necesario colocar el currículo en el centro de la acción evaluativa, rescatando sus niveles de concreción en el aula y la política educativa, y estableciendo para ello una articulación que no está puesta en sus aspectos eco-

2 En la legislación vigente, la atribución queda prácticamente idéntica, en lugar de considerar al INEE considerará a la Comisión. DOF, 2019. http://www.diputados.gob.mx/LeyesBiblio/pdf/LGE_300919.pdf

Diálopos 
nómicos, sociales o políticos — aunque sí condicionan la naturaleza del currículo—, sino en sus características didácticas y pedagógicas como espacio exclusivo de incidencia curricular. No obstante, lo urgente es establecer las sinergias entre estas otras disciplinas y campos de acción, que converjan no solo al interior del Instituto sino al exterior de este, para estar en posibilidad de construir evaluaciones técnicamente suficientes, con objetivos claros y con objetos de evaluación definidos que incluyan y reconozcan los abordajes didáctico-pedagógicos del currículo como acercamientos metodológicos y evaluativos de igual importancia a los otros que ocurren en el instituto.

\section{Lecciones aprendidas}

Como lo señalan Castro, Castro y Morales (2005), el proceso de investigación —y aquí añadimos el proceso de evaluación-, ante todo es un proceso creativo que, por tanto, debe estar abierto a su actualización, la cual se dará como producto de la propia actividad investigadora y en este caso la evaluativa. En ese sentido, recuperar la lógica de investigación ha significado la posibilidad de aplicar estrategias rigurosas pero a la vez flexibles, para construir evaluaciones asentadas en la realidad de las situaciones, procesos, sujetos y estructuras de las que trata de dar cuenta cada evaluación y trabajo presentado. De este modo y como conclusiones parciales, se presentan las siguientes lecciones aprendidas, mismas que se rescatan del marco referencial antes mencionado (INEE, 2019).

- Una mirada al desarrollo de la educación obligatoria en nuestro país muestra la fuerte apuesta depositada en el currículo como un eje articulador del cambio y la transformación educativa. En el contexto educativo mexicano, el currículo es un componente configurador de la realidad escolar; a partir de lo propuesto en él se definen normas administrativas, lineamientos $u$ orientaciones pedagógicas, y se generan materiales educativos, entre otros. Esto permite dar cuenta no solo de la complejidad que implica la evaluación del currículo de la educación obligatoria, sino también de la importancia de realizar esta tarea.

- El desafío de la evaluación del currículo está en desarrollar evaluaciones que permitan comprender la complejidad de la realidad educativa, al mismo tiempo que ofrezcan orientaciones puntuales acerca de lo que funciona bien o de lo que requiere atención. Implica tener claridad acerca de las fronteras de las dimensiones curriculares (diseño, implementación e impacto), pero sin perder de vista que en conjunto integran un todo y que cuando se interpela a alguna de estas, de manera indirecta se hace referencia a las otras. En ese sentido, será importante considerar diversas metodologías, áreas del instituto, instituciones y actores que permitan articular las evaluaciones de las tres dimensiones del currículo. 
- El diseño de nuevas propuestas curriculares para los niveles de la educación obligatoria debería considerar que su concreción en las escuelas y su ejecución por parte de los actores escolares requiere de algo más que buenas intenciones. Es necesario evaluar y dar seguimiento a su concreción en los planes y programas de estudio y materiales educativos asociados, así como también a su implementación en los espacios escolares. En el contexto educativo mexicano, las reformas curriculares usualmente están asociadas a los tiempos políticos, lo cual impone retos a la evaluación del currículo que no son triviales y que, sin lugar a dudas, pueden llegar a condicionar las tareas de evaluación.

- Antes de avanzar hacia allá, será necesario que los actores educativos tomen conciencia de la importancia que tiene el currículo y su evaluación, por lo que a mediano plazo se deberá aspirar a que las autoridades educativas y escolares identifiquen la evaluación del currículo como una forma de contribuir a la mejora de la educación. En ese sentido, es importante que reconozcan que evaluar el currículo es un proceso permanente que exige cuando menos dos condiciones: la participación de los actores involucrados en el diseño y puesta en marcha del currículo, y que el resultado de estas evaluaciones sea oportuno y se utilice para incidir de manera efectiva en el desarrollo del currículo.

- Se requiere impulsar y consolidar la evaluación curricular como un proceso fundamental que imbrica otros componentes del SEN. Para ello es necesario articular las evaluaciones curriculares con otras que realiza el instituto, establecer proyectos que deriven en evaluaciones más integrales en los que intervengan diversas áreas del instituto y otras instancias educativas que participan en los diferentes procesos curriculares; así como abrir espacios para intercambiar conocimiento sobre este campo.

Cabe señalar que todos estos puntos se lograrán a partir de un uso pertinentes y riguroso de la metodología de investigación como medio para producir conocimiento, identificar espacios problemáticos, así como contribuir a su valoración, que es el propósito final de la evaluación, para impulsar cambios que sean traducibles al currículo y a las prácticas que desde aquí se despliegan.

\section{Referencias}

Becher, T. (2009). Tribus y territorios académicos: la indagación intelectual y la cultura de las disciplinas. España: Gedisa.

Camilloni, A. (2007). Justificación de la didáctica. En Camilloni, A. (comp.). El saber didáctico. Argentina: Paidós Educación.

Castro, L., M. Castro y J. Morales (2005). Metodología de las ciencias sociales. Una introducción crítica. España: Tecnos. 
Daviet, B. (2016). Revisar el principio de la educación como bien público. Documentos de Investigación y Prospectiva en Educación, 17. París: UNESCO

De Alba, A. (1991). Currículo, crisis, mito y perspectiva. México: UNAM.

De Ibarrola, M. (2012). Experiencias y reflexiones sobre el diseño y la evaluación curricular, en M. Landesmann (coord.) El currículum en la globalización. A tres décadas de "el currículum pensado y el currículum vivido", México: UNAM-FES Iztacala, 93-118.

(2006). La formación para el trabajo en las escuelas: el currículo y las prácticas formativas. En M. De Ibarrola, Formación escolar para el trabajo. Posibilidades y límites. Montevideo: CINTERFOR/OIT, 151-210.

(1985). Las dimensiones sociales de la educación. México: SEP/El Caballito.

De la Rosa, A. (2002), Teoría de la organización y nuevo institucionalismo en el análisis organizacional. Administración y Organizaciones, 4(8), 2002, 13-44.

Díaz, J. (2005). Modelo científico: conceptos y usos. En López Austin, A. (coord.). El modelo en la ciencia y la cultura. México: UNAM/Siglo XXI, 11-28.

Díaz Barriga, A. (coord.) (2013). La investigación curricular en México, 2002-2011. México: COMIE/ ANUIES.

(1995). La evaluación curricular. Una aproximación a elementos centrales de esta disciplina y su ubicación en México. Cuadernos Pedagógicos Universitarios, (8), 1-37.

DOF (2013) Ley General de Educación. México. https://www.sep.gob.mx/work/models/sep1/ Resource/558c2c240b12-4676-ad90-8ab78086b184/ley general educacion.pdf

Dussel, I. (2014) ¿Es el curriculum escolar relevante en la cultura digital? Debates y desafíos sobre la autoridad cultural contemporánea. Archivos Analíticos de Políticas Educativas, 22(24). http://dx.doi.org/10.14507/epaa.v22n24.2014

Elster, J. (2005) En favor de los mecanismos. Revista Sociológica, 57, 239-273.

Galindo, J (2012). Entre la necesidad y la contingencia: Autoobservación teórica de la sociología. México: Anthropos/UAM-Cuajimalpa.

García, J. (2014) Estudio Introductorio. En W. Pinar, La teoría del currículum. Madrid: Narcea.

García, R. (2001). Fundamentación de una epistemología en ciencias sociales. Estudios Sociológicos, XIX(57), 615-620.

Gimeno Sacristán, J. (2011). ¿Qué significa el currículum? En J. Gimeno Sacristán, R. Feito, P. Perrenoud y M. Clementa (coords.) Diseño, desarrollo e innovación del currículum. Madrid: Editorial Morata.

(2007). El currículum: una reflexión sobre la práctica. España: Morata.

Guzmán G., C. (2018). Avances y dificultades en la implementación del Marco Curricular Común. Telebachillerato estatal, Educación Media Superior a Distancia y Telebachillerato comunitario. México: INEE.

Habermas, J. (1987). La idea de universidad-procesos de aprendizaje. Sociológica, 2(5), 2-18. 
Hernández, L., J. Romero y N. Bracho (2005). Tesis básicas del racionalismo crítico. Cinta Moebio, 23, 193-203. www.moebio.uchile.cl/23/hernandez.htm

Hernández Sampieri, R., C. Fernández Collado y P. Baptista Lucio (2010). Metodología de la investigación. México: McGrawHill.

Kuhn, T. (2013) La estructura de las revoluciones científicas. México: FCE.

INEE (2016). La educación obligatoria en México. Informe 2016. INEE. México. https://www.inee. edu.mx/wp-content/uploads/2018/12/P1/241.pdf

INEE (2018). La implementación del Marco Curricular Común en los planteles de la educación media superior. México. https://www.inee.edu.mx/wp-content/uploads/2018/12/P1D248. pdf

INEE (2019). Análisis del diseño del componente de autonomía curricular. Plan y programas de estudio 2017. México: INEE. https://www.inee.edu.mx/wp-content/uploads/2019/05/P1F231. pdf

INEE (2019). Evaluación del currículo de educación ciudadana en la educación obligatoria. México. https://www.inee.edu.mx/wp-content/uploads/2019/08/P1F232.pdf

INEE (2019). Informe de resultados de EIC-Preescolar 2017: creencias de las figuras docentes sobre el currículo, la enseñanza y el aprendizaje. México: INEE. https://www.inee.edu.mx/wp-content/uploads/2019/05/ResultadosEIC-Preescolar2017.pdf

INEE (2019). Orientaciones técnicas para la evaluación del diseño curricular. Evaluación de contenidos y métodos educativos. México: INEE. https://www.inee.edu.mx/wp-content/ uploads/2019/05/P1E217.pdf

INEE (2019). Marco referencial y metodológico para la evaluación de contenidos y métodos educativos. México. https://www.inee.edu.mx/wp-content/uploads/2019/01/P1E211.pdf

IJiménez García, S. (2012). Investigadores experimentados, sus nociones de formación, en N. Gutiérrez Serrano (coord.). Formación, política e investigación. Espacios de producción de conocimiento en educación en México y el Cono Sur. México: CRIM/UNAM/Díaz de Santos.

Massé, C. (2003). De la lógica de la investigación a la lógica como objeto. Un esbozo de las cuestiones centrales del racionalismo crítico de K. Popper. Antropología Experimental, (3), 1-25. http://revista.ujaen.es/huesped/rae/articulos2003/massenarvaez2003.pdf

Muñoz Izquierdo, C. (2003). Desarrollo de una propuesta para la construcción de indicadores del impacto social de la educación en América Latina y el Caribe. México: UIA.

Perrenoud, P. (2011). El "currículum real" y el trabajo escolar. En Gimeno Sacristán, J., R. Feito, P. Perrenoud y M. Clementa (coords.). Diseño, desarrollo e innovación del currículum. Madrid: Editorial Morata.

Pinar, W. (2003). International handbook of curriculum research. USA: Lawrence Erlbaum Associates. http://www.khuisf.ac.ir/dorsapax/userfiles/file/motaleat/080583222x.pdf 
Ramírez, J. (2000). Teoría de la organización: metáforas y escuelas. En Arellano, D., E. Cabrero, y A. Del Castillo (coords.). Reformando al gobierno: una visión organizacional del cambio gubernamental. México: Miguel Ángel Porrúa/CIDE.

Reynoso Angulo, R. y Chamizo Guerrero, J. A. (coords.) (2017). Estudio comparativo de la propuesta curricular de ciencias en la educación obligatoria en México y otros países. México: INEE.

Rojano Ceballos, M. T. y Solares Rojas, A. (coords.) (2017). Estudio comparativo de la propuesta curricular de matemáticas en la educación obligatoria en México y otros países. México: INEECinvestav.

Schmelkes, S. (2001). La combinación de estrategias cuantitativas y cualitativas en la investigación educativa: reflexiones a partir de tres estudios. REDIE, Revista Electrónica de Investigación Educativa, 3(2), Universidad Autónoma de Baja California, México.

Stigler, J. y Hierbert, J. (2002). La brecha en la enseñanza. Estudios Públicos, 86, 57-144. https:// www.cepchile.cl/cep/site/artic/20160304/asocfile/20160304092948/rev86 stigler.pdf

Stabback, P. (2016). ¿Qué hace a un currículo de calidad? Reflexiones en progreso. UNESCO.

Weiss H., E. (2017). Estudio exploratorio del Modelo de Telebachillerato Comunitario y su operación en los estados. México: INEE.

Weiss, E. y Gutiérrez Serrano, N. (2009). Introducción, en N. Gutiérrez Serrano (coord.). Redes, comunidades, grupos y trabajo entre pares en la Investigación Educativa. México: CRIM-UNAM/ Plaza y Valdés Editores. 